\title{
Effect of diazepam on myocardial blood flow of patients with and without coronary artery disease
}

\author{
Hamid Ikram, Anthony P. Rubin, and Reginald F. Jewkes \\ From Charing Cross Hospital, London
}

Myocardial blood flow was measured by the ${ }^{133} \mathrm{Xe}$ clearance method in 6 patients with radiologically confirmed disease of the coronary arteries and in 4 patients with normal arteries. The myocardial blood flow was measured 5 minutes after intravenous diazepam $0.1 \mathrm{mg} / \mathrm{kg}$ body weight. There was an increase in myocardial blood flow in all cases. Patients with normal coronary arteries showed an average increase of 22.5 per cent over control levels while patients with diseased coronary arteries showed a 73 per cent average increase. Both figures were highly significant statistically. Possible mechanisms and implications of these findings are discussed.

The benzdiazepine family of drugs (chlordiazepoxide, diazepam, oxazepam, and medazepam) is widely used for producing mild sedation and relieving emotional tension. Diazepam is very popular in cardiological practice. It has been used for premedication before cardiac catheterization (Tornetta, 1965) and for producing analgesia/amnesia for DC 'cardioversion' (Nutter and Massumi, 1965; Somers et al., I97I).

The tranquillizing properties of diazepam have been used in the management of patients with coronary artery disease. The rationale for this lies in the common clinical experience that anginal pain is often precipitated or exacerbated by emotional upset, and that relieving this results in improvement.

The mechanism by which emotion produces angina is poorly understood, but is presumed to be due to the action of higher centres mediated via the autonomic nervous system. The beneficial action of tranquillizing drugs is thought to be due to their action on the psyche.

However, there is a certain amount of evidence from animal studies that diazepam increases coronary blood flow in the isolated canine heart preparation (Abel, Reis, and Staroscik, I970a, b; Taylor, Daniell, and Bagwell, 1970). As far as we are aware, there is no information on the action of this drug on human myocardial blood flow.

The purpose of this paper is to report the effects of intravenous diazepam on myocardial blood flow in patients with normal and diseased coronary arteries.

Received 3 August 1972.

\section{Subjects and methods}

Studies were made on Io patients, 6 with clinical coronary artery disease and 4 with valvular heart disease. Cardiac catheterization was performed for evaluation before operation. The patients were unpremedicated and were studied after an overnight fast.

In 8 patients, myocardial blood flow was measured by the Xenon ${ }^{133}$ clearance method of Ross et al. (1964) as modified in this laboratory (Ikram et al., I973).

The left main coronary artery was selectively catheterized by the Judkins technique (Judkins, 1967). This vessel was chosen for study because stable cannulation can easily be performed by the Judkins method. Furthermore, since the portion of the myocardium supplied by it drains into the coronary sinus, myocardial blood flow measurement by techniques based on coronary sinus sampling measure left coronary artery flow. The relevance of this to the study will be explained subsequently. Cannulation of the coronary artery was confirmed by fluoroscopic observations of injected contrast medium, 76 per cent Renograffin. In order to minimize haemodynamic changes due to the contrast medium, it was diluted with an equal volume of normal saline, and a delay of ro minutes was allowed before measurements of myocardial blood flow.

${ }_{400-500 \mu} \mu \mathrm{Ci}{ }^{133} \mathrm{Xe}$ in saline solution were injected into the left coronary artery and flushed through with $8 \mathrm{ml}$ normal saline. The catheter was removed from the coronary ostium immediately after completion of the injection. The activity was monitored by a Pitman detector positioned fluoroscopically over the centre of the cardiac shadow. The detector consisted of a collimated sodium iodide crystal connected to a batterypowered ratemeter with an integration constant of 2 seconds. The change in radioactivity with time (Fig. I) was recorded on an SE Labs 3006 U.V. Recorder.

Two control measurements were made after which 

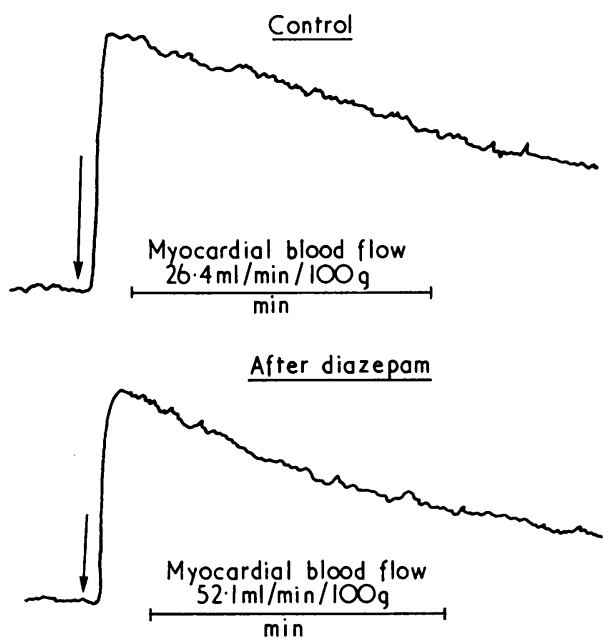

FIG. I ${ }^{133} \mathrm{Xe}$ myocardial clearance curves. Control (above) and after intravenous diazepam (below).

diazepam in a dose of $0.1 \mathrm{mg} / \mathrm{kg}$ body weight was injected via a catheter in the right atrium. Myocardial blood flow was measured 5 minutes after the injection of diazepam.

Myocardial blood flow was calculated from the equation,

Myocardial blood flow $(\mathrm{ml} / \mathrm{min} / \mathrm{IO0} \mathrm{g})=\frac{\log _{\mathrm{e}} 2 \times \lambda \times \mathrm{IO0}}{\mathrm{T} \frac{1}{2} \times \mathrm{P}}$ where

$\lambda=$ partition coefficient of Xenon in blood and myocardium $=0.72$

$\mathrm{P}=$ density of the myocardium $=\mathrm{I} .05$

$T \frac{1}{2}=$ time taken by the exponentially decaying time activity curve to fall from any value to half that value. This is obtained from a semilogarithmic plot of activity against time (Fig. 2) and is the only measurement made from the curve.

In 2 patients the technique described by Cohen, Elliott, and Gorlin (1964) was used to measure myocardial blood flow using ${ }^{133} \mathrm{Xe}$ instead of ${ }^{85} \mathrm{Kr}$ as the test substance. This method required cannulation of the coronary sinus. $500 \mu \mathrm{Ci}^{133} \mathrm{Xe}$ were injected into the left ventricle. Coronary sinus blood was sampled at Iminute intervals, starting $45 \mathrm{sec}$ after injection. The activity in each sample was measured by means of a bench well counter. A log-linear plot of activity against time was constructed and $T \frac{1}{2}$ measured from this. Myocardial blood flow was calculated by substitution in the same equation as before.

This technique for measurement of myocardial blood flow was utilized to make certain that instrumentation of the coronary artery and injection of contrast medium was not producing spurious results as it does not involve entry or injection into the vessel.

After completion of myocardial blood flow measurements, selective coronary angiography was performed by injection of 76 per cent Renografin and filming this on cine film at 48 frames $/ \mathrm{sec}$.

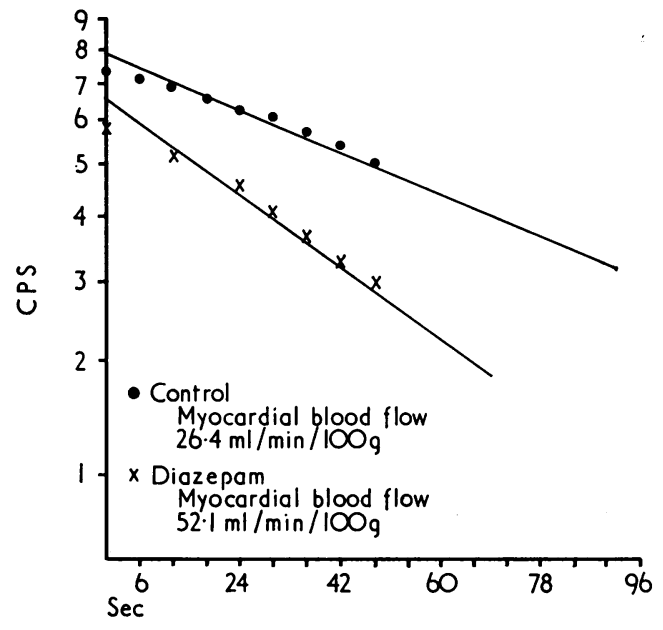

FIG. 2 Log-linear plot of curves in Fig. I. Radioactivity along logarithmic scale and time along linear scale.

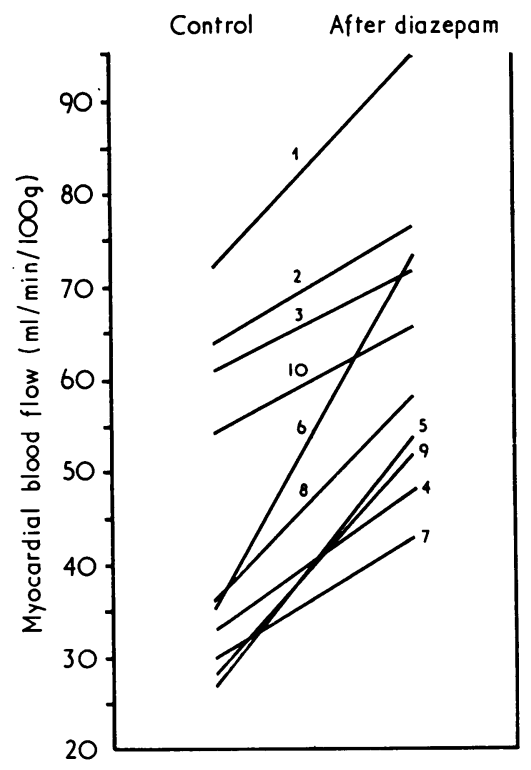

FIG. 3 Myocardial blood flow before and after diazepam.

\section{Results}

The results are summarized in the Table and Fig. 3. Statistical analysis was performed by standard parametric methods, the significance of the various mean results being evaluated by the Student ' $t$ ' test.

The mean control myocardial blood flow for patients with coronary artery disease was 35.55 
TABLE Patients with and without coronary artery disease

\begin{tabular}{|c|c|c|c|c|}
\hline \multirow{2}{*}{$\begin{array}{l}\text { Case } \\
\text { No. }\end{array}$} & \multirow[t]{2}{*}{ Diagnosis } & \multicolumn{2}{|c|}{ Myocardial blood flow ( $\mathrm{mg} / \mathrm{min} / \mathrm{roo} \mathrm{mg}$ ) } & \multirow{2}{*}{$\begin{array}{l}\text { Per cent } \\
\text { change }\end{array}$} \\
\hline & & Control & After diazepam & \\
\hline \multicolumn{5}{|c|}{ Patients with coronary artery disease } \\
\hline 4 & Old anterior infarct, left anterior descending artery occlusion & $\left.\begin{array}{l}34 \cdot 9 \\
30 \cdot 7\end{array}\right\} 32 \cdot 8$ & $48 \cdot 0$ & +46 \\
\hline 5 & $\begin{array}{l}\text { Multiple narrowing of left coronary artery with 'ischaemic' } \\
\text { cardiomyopathy }\end{array}$ & $\left.\begin{array}{l}24 \cdot I \\
30 \cdot 2\end{array}\right\} 27 \cdot 2$ & $53 \cdot 6$ & +97 \\
\hline 6 & Left circumflex block & $\left.\begin{array}{l}31 \cdot 3 \\
39 \cdot 2\end{array}\right\} 35 \cdot 2$ & $73 \cdot 1$ & +107 \\
\hline 7 & Left anterior descending artery block & $\left.\begin{array}{l}32 \cdot 1 \\
27 \cdot 8\end{array}\right\} 29 \cdot 9$ & $43 \cdot 3$ & +44 \\
\hline $8^{\star}$ & Left anterior descending artery narrowed, mitral valve disease & $35 \cdot 7$ & $57 \cdot 9$ & +62 \\
\hline \multirow[t]{2}{*}{9} & Left circumflex block & $\left.\begin{array}{l}30 \cdot 6 \\
26 \cdot 4\end{array}\right\} 28 \cdot 5$ & $52 \cdot 1$ & +82 \\
\hline & Mean & $31 \cdot 55$ & $54 \cdot 66$ & $+73 \%$ \\
\hline \multicolumn{5}{|c|}{ Patients without coronary artery disease } \\
\hline $\mathbf{I}$ & Leaking aortic Starr valve & $\left.\begin{array}{l}74 \cdot 1 \\
70 \cdot 0\end{array}\right\} 72$ & $94 \cdot 6$ & $+3 I$ \\
\hline 2 & Aortic stenosis and incompetence & $\left.\begin{array}{l}69 \cdot 9 \\
57 \cdot 3\end{array}\right\} 63 \cdot 6$ & $76 \cdot 6$ & +20 \\
\hline 3 & Mild aortic stenosis & $\left.\begin{array}{l}59 \cdot 4 \\
64 \cdot 1\end{array}\right\} 60 \cdot 7$ & $72 \cdot 3$ & +19 \\
\hline \multirow[t]{2}{*}{ I0* } & Mitral stenosis & $54 \cdot 2$ & 65 & +19 \\
\hline & Mean & $62 \cdot 6$ & $77 \cdot 1$ & $+22 \cdot 5 \%$ \\
\hline
\end{tabular}

* Myocardial blood flow by method of Cohen et al. (1964).

$\mathrm{ml} / \mathrm{min} / 100 \mathrm{~g}$ compared to $62.60 \mathrm{ml} / \mathrm{min} / 100 \mathrm{~g}$ for patients with a radiologically normal left coronary artery $(\mathbf{P}<0.0005)$. After administration of diazepam, the myocardial blood flow increased in patients with and without coronary artery disease.

In patients with coronary artery disease the myocardial blood flow after diazepam was $54.66 \mathrm{ml} / \mathrm{min} /$ roo $\mathrm{g}$, which represents a significant increase $(\mathrm{P}<0.0025)$.

In patients without coronary artery disease, the myocardial blood flow increased to $77.1 \mathrm{ml} / \mathrm{min} / 100$ $\mathrm{g}(\mathbf{P}<0.02)$.

The percentage increase in patients with coronary artery disease was 73 compared to 22.5 in patients without coronary artery disease. The difference was significant at a level of $P<0.005$.

\section{Discussion}

Our results show that diazepam increases coronary blood flow in man just as it has been shown to do in the isolated canine heart (Abel et al., I970a, b; Taylor et al., 1970).

Abel et al. (1970a) studied the effects of diazepam in a dose of $0.1 \mathrm{mg} / \mathrm{kg}$ body weight on the myocardial blood flow of 7 normal dogs in whom coronary perfusion and heart rate were held constant. In each case the coronary blood flow increased, the average increase being 14.8 per cent in 35-50 sec after administration of diazepam. These authors attributed the increased myocardial blood flow to the fall in coronary vascular resistance which they observed. Since these changes also occurred in the isolated heart they concluded that this could not be due to extracardiac humoral materials or to reflexes mediated via the effect of diazepam on the central nervous system.

In another study, Abel et al. (1970b) suggested a hypothesis to explain the coronary vasodilatation produced by diazepam. The hypothesis is based on the neurophysiological studies of Szentivanyi and Juhasz-Nagy (1959) and Juhasz-Nagy and Szentivanyi (196I) on the canine heart. They showed that there were three types of autonomic nerves which supply the canine heart. One type passes through the cervical ganglion and synapses with ganglia in the coronary arteries or myocardium. Stimulation 
of these nerves, which are cholinergic, produces coronary vasodilatation. Abel et al. (1970b) found that diazepam-induced coronary vasodilatation was completely opposed by ganglion blockade with trimetaphan. On the basis of these experiments they concluded that diazepam caused coronary vasodilatation by specifically stimulating vasodilator mechanisms at the postganglionic neurone.

The average increase of 22.5 per cent observed in our patients with normal coronary arteries is similar to the 14.8 per cent increase observed in the normal dog. The mechanism by which these changes are produced in human beings was not investigated by us, since to do so would involve ethically unjustified instrumentation and autonomic blockade in order to produce meaningful results. Furthermore, the autonomic innervation of the human coronary arterial system is so poorly understood as to make any interpretation highly speculative. We assume that the mechanisms producing the increased myocardial blood flow are probably similar to those in the dog heart, namely coronary vasodilatation mediated by the autonomic nervous system.

The increase in myocardial blood flow is not secondary to alteration in systemic haemodynamics, since in a previous study we found that intravenous diazepam in this dose did not produce any significant change in systemic arterial pressure, cardiac output, or right ventricular dp/dT (Ikram and Rubin, I97I).

The response of subjects with coronary artery disease to diazepam is of interest. These subjects showed a much greater response to diazepam, suggesting that the coronary vascular bed behaves differently in this condition. Though changes in human myocardial blood flow are difficult to interpret, there is a certain amount of evidence which suggests that coronary vasomotor responses are abnormal in patients with coronary artery disease. Messer et al. (1963) compared the effects of exercise in patients with coronary disease to those in normal subjects; the patients showed a greater increase in coronary blood flow at the same level of exercise. Hepps, Roe, and Rutkin (1963) studied the effects of nitroglycerin in the coronary blood flow of dogs. In the control period, intravenous nitroglycerin only caused a transient increase in coronary blood flow. However, if coronary flow was previously decreased by thyrotrophin, a much more prolonged and pronounced increase was produced.

The differing results obtained for myocardial blood flow in patients with normal and diseased coronary arteries are presumably due to the influence of collateral vessels. Harrison and Reeves (I968) offer an attractive hypothesis to explain the often conflicting results of the action of coronary vasoactive drugs. They state that in the normal myo- cardium the chief site of myocardial vascular resistance lies in the small intramural vessels. Their calibre is governed chiefly by local metabolites and not by the autonomic nerves. In patients with coronary artery disease the intramural vessels are occluded and blood flows to the ischaemic area via collaterals which then become the vessels determining myocardial resistance. Since they are derived from the medium-sized branches of the coronary arteries, they are very susceptible to changes in autonomic function. Commenting on this role of collateral vessels in the genesis of anginal attacks, Harrison and Reeves (I968) state that, 'there is evidence that in at least some instances, anginal attacks are initiated by constriction or reduced dilatation of coronary arteries, and perhaps especially of previously widened collaterals'.

The mere fact that diazepam increases myocardial blood flow is not necessarily synonymous with a beneficial therapeutic effect in angina. The deciding factor seems to be whether the increased flow is delivered to the ischaemic area or diverted to the healthy portions of the myocardium. This problem is exemplified by the actions of two coronary vasodilator drugs, nitroglycerin and dipyridamole. Nitroglycerin relieves anginal pain since it acts on the proximal large coronary arteries and the collaterals derived from these, which supply the ischaemic area. In contrast, dipyridamole dilates the small intramural vessels. These are blocked in the ischaemic area so coronary blood flow is diverted to healthy portions of the myocardium, accounting for the lack of benefit of this drug in angina (Fam and McGregor, 1964; Fam, Nelligan, and McGregor, 1966). Increased delivery of blood to ischaemic areas rather than an increase in total coronary blood flow appears to determine whether a drug is effective in angina.

The question of therapeutic efficacy can only be resolved by a controlled clinical trial, but this study demonstrates a direct cardiac effect by diazepam, which, rather than the psychological effects, may explain its observed beneficial action in angina. Many anxiety-relieving drugs used in the treatment of ischaemic heart disease are related to diazepam or are known vasodilators, e.g. chlorpromazine. In view of this, theories on the role of the psyche in ischaemic heart disease based on the therapeutic response to these drugs seem to require re-evaluation.

A point of practical importance is that diazepam is often given to patients in controlled clinical trials of antianginal drugs. In view of its coronary vasodilator action it seems wise not to administer this type of drug or to alter its dose during such clinical trials. 
The authors wish to express their gratitude to Mr. A. Bartoli, Miss C. S. March, and Miss G. Chatsfield for technical assistance. The Clinical Research Sub-Committee of Charing Cross Hospital provided generous financial support.

\section{References}

Abel, R. M., Reis, R. L., and Staroscik, R. N. (I970a). Coronary vasodilation following diazepam (Valium). British fournal of Pharmacology, 38, 620 .

Abel, R. M., Reis, R. L., and Staroscik, R. N. (1970b). The pharmacological basis of coronary and systemic vasodilator actions of diazepam (Valium). British fournal of Pharmacology, 39, 26r.

Cohen, L. S., Elliott, W. C., and Gorlin, R. (1964). Measurement of myocardial blood flow using krypton-85. American Fournal of Physiology, 206, 997.

Fam, W. M., and McGregor, M. (1964). Effect of coronary vasodilator drugs on retrograde flow in areas of chronic myocardial ischaemia. Circulation Research, 15, 355.

Fam, W. M., Nelligan, D., and McGregor, M. (I966). Nitroglycerine and dipyridamole: two vasodilators with different sites of action in the coronary vascular tree (abstract). Circulation, 34, Suppl. III, 100.

Harrison, T. R., and Reeves, T. J. (1968). The Principles and Problems of Ischaemic Heart Disease, pp. 248-249. Year Book Medical Publishers, Chicago.

Hepps, S. A., Roe, B. B., and Rutkin, B. B. (1963). Coronary blood flow in the intact conscious dog: studies with miniature electromagnetic flow transducers. Fournal of Thoracic and Cardiovascular Surgery, 46, 783.

Ikram, H., and Rubin, A. P. (197I). Anaesthesia for cardioversion. British Medical fournal, 4, 626.
Ikram, H., Rubin, A. P., Jèwkes, R. F., and Bartoli, A. (1973). The measurement of selective myocardial blood flow by $\mathrm{Xe}^{133}$ clearance method using a portable battery-powered ratemeter and scintillation counter. Biochemical Engineering. In the press.

Judkins, M. P. (1967). Selective coronary arteriography. A percutaneous transfemoral technic. Radiology, 89, 815.

Juhasz-Nagy, A., and Szentivanyi, M. (196I). Separation of cardioaccelerator and coronary vasomotor fibres in the dog. American fournal of Physiology, 200, 125.

Messer, J. V., Levine, H. J., Walman, R. J., and Gorlin, R. (1963). Effects of exercise on cardiac performance in human subjects with coronary artery disease. Circulation, 28, 404 .

Nutter, D. O., and Massumi, R. A. (1965). Diazepam in cardioversion. New England fournal of Medicine, 273, 650.

Ross, R. S., Ueda, K., Lichtlen, P. R., and Rees, J. R. (1964). Measurement of myocardial blood flow in animals and man by selective injection of radioactive inert gas into the coronary arteries. Circulation Research, 15, 28.

Somers, K., Gunstone, R. F., Patel, A. K., and D'Arbela, P. G. (I97I). Intravenous diazepam for direct-current cardioversion. British Medical fournal, 4, 13.

Szentivanyi, M., and Juhasz-Nagy, A. (1959). A new aspect of the nervous control of the coronary blood vessels. Quarterly fournal of Experimental Physiology, 44, 67.

Taylor, P. L., Daniell, H. B., and Bagwell, E. E. (1970). Studies on the myocardial hemodynamic and metabolic effects of diazepam in the dog (abstract). Pharmacologist, 12, 233.

Tornetta, F. J. (1965). Diazepam as pre-anaesthetic medication. Anesthesia and Analgesia, 44, 449.

Requests for reprints to Dr. H. Ikram, Charing Cross Hospital, London $\mathrm{WC}_{2} \mathrm{H}{ }_{4} \mathrm{DZ}$. 